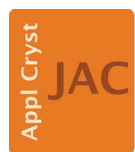

JOURNAL OF

APPLIED

CRYSTALLOGRAPHY

ISSN 1600-5767

Received 4 April 2017

Accepted 4 April 2017

Keywords: crystal forms; systematic absences; Miller indices; symmetry restrictions; crystallographic education.

\section{The ash heap of crystallography: restoring forgotten basic knowledge. Corrigendum}

\author{
Massimo Nespolo* \\ Université de Lorraine, CRM2, UMR 7036, Vandoeuvre-les-Nancy, F-54506, France, and CNRS, CRM2, UMR 7036, \\ Vandoeuvre-les-Nancy, F-54506, France. *Correspondence e-mail: massimo.nespolo@univ-lorraine.fr
}

One imprecise and one incomplete statement in the article by Nespolo [J. Appl. Cryst. (2015), 48, 1290-1298] are corrected.

In our article (Nespolo, 2015), one imprecise and one incomplete statement about the conditions for Miller indices to be relatively prime have been identified, which we correct hereafter.

Consider the statement on page 1293, after equation (2): 'When the unit cell is primitive or when the planes of the $(h k l)$ family are parallel to the face of the unit cell containing the centring vector, the above condition is consistent with coprime Miller integers.' In that sentence, the term 'the centring vector' should be replaced by 'a centring vector'. In the example given for the $o C$ unit cell in Fig. 1 therein, centring vectors go from the origin to the lattice nodes $\frac{1}{2} \frac{1}{2} 0, \frac{1}{2} \frac{1}{2} 0, \frac{1}{2} \frac{1}{2} 0$ and $\frac{1}{2} \frac{1}{2} 0$. These are all contained in the (001) plane, but only two of them, namely $\frac{1}{2} \frac{1}{2} 0$ and $\frac{1}{2} \frac{1}{2} 0$, are contained in the (110) plane.

On the same page, equation (2),

$$
h x+k y+l z=n,
$$

is followed by the statement 'it should give the first plane of the family after the origin, in the positive direction, when $n=$ 1 ', which should be completed by the condition 'and $n$ is minimal'. For example, in the case of the plane (301), all the

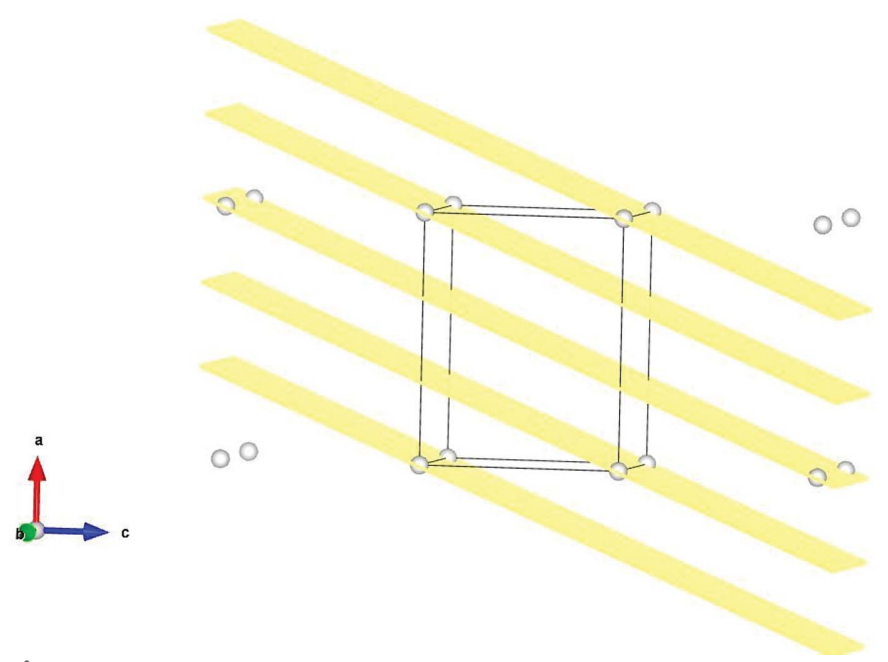

Figure 1

The (301) family of lattice planes for a primitive unit cell. From bottom to top of the figure, the planes correspond to $n=0,1,2,3,4$ in equation (2). 




Figure 2

The same family of lattice planes for a $C$-centred unit cell. Planes shown in orange do not exist in a primitive unit cell. If the same Miller indices, (301), are used, then these planes would correspond to half-integer values of $n$ in equation (2) and the one leading to $n=1$ is not the first plane of the family but the second one, contrary to the definition of Miller indices. Accordingly, from bottom to top of the figure, the planes would correspond to $n=0, \frac{1}{2}, 1, \frac{3}{2}, 2, \frac{5}{2}, 3, \frac{7}{2}, 4$ in equation (2). By adopting the correct non-relatively prime indices (602), the first plane of the family does correspond again to $n=1$ in equation (2); from bottom to top of the figure, the planes correspond now to $n=0,1,2,3,4,5,6,7,8$. Figures drawn with VESTA (Momma \& Izumi, 2011). planes of the family are parallel to the $b$ axis. If the unit cell is primitive, the three first planes have intercepts on the $a$ and $c$ axes at $x=\frac{1}{3}, z=1 ; x=\frac{2}{3}$ and $z=2$; and $x=1$ and $z=3$, respectively (Fig. 1). Accordingly, they pass through lattice nodes $0 v 1,0 v 2$ and $1 v 0$ (any integer $v$ ), which leads to $n=1,2$ and $n=3$ in equation (2). In this case, (301) are the correct Miller indices. If the unit cell is $C$ centred, however, the first three planes for the family have intercepts $x=\frac{1}{6}, z=\frac{1}{2} ; x=\frac{1}{3}$, $z=1 ; x=\frac{1}{2}, z=\frac{3}{2}$ (Fig. 2). Accordingly, they pass through lattice nodes $\frac{1}{2}\left(v+\frac{1}{2}\right) 1,0 v 1$ and $\frac{1}{2}\left(v+\frac{1}{2}\right) 0$ (any integer $v$ ), which leads to $n=0.5,1$ and $n=1.5$, respectively. Because the minimal value of $n$ is not 1, the Miller indices of the family are not (301) but (602).

\section{Acknowledgements}

We thank Professor Carolyn P. Brock (University of Kentucky, USA) for pointing out the incorrect statements.

\section{References}

Momma, K. \& Izumi, F. (2011). J. Appl. Cryst. 44, 1272-1276. Nespolo, M. (2015). J. Appl. Cryst. 48, 1290-1298. 


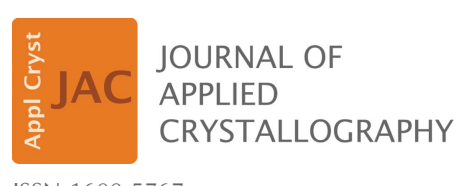

ISSN 1600-5767

Received 17 February 2015

Accepted 9 June 2015

Edited by J. M. García-Ruiz, Instituto Andaluz de Ciencias de la Tierra, Granada, Spain

₹ On leave from Université de Lorraine, Faculté des Sciences et Technologies, Institut Jean Barriol FR 2843, CRM2 UMR CNRS 7036, BP 70239, Boulevard des Aiguillettes, F-54506

Vandoeuvre-lès-Nancy Cedex, France.

Keywords: crystal forms; systematic absences; Miller indices; symmetry restrictions; crystallographic education.

\section{The ash heap of crystallography: restoring forgotten basic knowledge}

\author{
Massimo Nespolo*¥ \\ Materials and Structures Laboratory, Tokyo Institute of Technology, Midori-ku, Yokohama City, Kanagawa 226-8503, \\ 4259 Nagatsuta, Japan. *Correspondence e-mail: massimo.nespolo@crm2.uhp-nancy.fr
}

A critical analysis of some basic notions often overlooked in crystallographic education is presented to correct some common oversights occurring both in the literature and in textbooks. The crystal forms (face forms), defined in terms of their geometric eigensymmetry, are 47 in number, not 48 as often found in the literature. The split of the dihedron into dome and sphenoid calls for the consideration of the physical properties of the faces building a form; in that case, however, the same criterion should be used for all forms. By taking the handedness of the faces as representative of the physical properties of the faces, the occurrence of 130 crystallographic face forms ( 97 affine face forms and 33 enantiomorphic pairs) is demonstrated. Next, the correct use of non-coprime Miller indices when a centred unit cell is adopted is shown, and the inconsistent multiplication of Miller indices in the Bravais-Friedel-Donnay-Harker law is pointed out. A geometric derivation of the reflection conditions is reviewed. Finally, the inconsistent presentation of metric restrictions imposed by the structural symmetry is pointed out and corrected.

\section{Introduction}

In 1836, the Italian poet Giacomo Leopardi (1836) ironically challenged what he called the 'magnificent and progressive fate of the human race', meaning the blind belief in an unlimited and extraordinary progress for all mankind, the optimistic legacy of the Age of Enlightenment. Two centuries later we realize how far-sighted was his scepticism: the undeniable technological advances and the evolution of scientific knowledge have not been paralleled by a comparable social evolution in many parts of our world. Scientific advances have increased exponentially the number and complexity of notions and concepts that one is expected to master in any field, so that some domains are increasingly being overlooked and considered of lower priority, and the corresponding education is continually reduced, sometimes to a minimal set of practical notions. Particularly affected by this tendency are the most interdisciplinary subjects, which belong to everywhere but nowhere specifically. Crystallography is one perfect example: it is tightly connected to all domains dealing with the structure and properties of periodic matter (in whatever dimensions and whatever geometry), spanning thus mineralogy (from which it was born at the dawn of modern scientific thinking), materials science, metallurgy, physics, chemistry, biochemistry and mathematics, but one would have a tough task if today one wanted to find a fully crystallographic lecture within a standard university curriculum. Some aspects of crystallography, often reduced to a minimal set of indispensable concepts, are dispersed in lectures specific to the major subjects. As a consequence, approximate, imprecise and sometimes fundamentally wrong statements and definitions appear not only in 
lectures but also in textbooks, which go to press without passing through the necessary screening of a competent crystallographer, who may well become an endangered species. The adoption of less effective presentations of basic concepts is also frequent today, while older and too often forgotten textbooks contain more effective and convincing approaches. By paraphrasing Leopardi's poem, one could say that the 'magnificent and progressive fate of crystallography', which one might expect as a legacy of the International Year of Crystallography celebrated last year, is jeopardized by the serious oversight and forgetting of many fundamental concepts which represent the roots of our discipline.

Without the ambition of an extensive analysis, a task which would require a much greater space and time frame, we dare to present in the following a few examples of the imprecisions, approximations and even mistakes often occurring in the literature, which we hope may awaken interest in filling some holes in the crystallographic background of those who have undertaken the noble yet hard mission of training future generations of knowledgeable crystallographers.

\section{On the number of face forms}

In crystallography, a form is defined as the set of all symmetrically equivalent 'elements' with respect to the point group $P$ of the crystal. The definition of 'element' depends on the space under consideration, namely

(i) in vector space, a crystal form or face form is a set of all symmetrically equivalent faces;

(ii) in point space, a point form is a set of all symmetrically equivalent points.

The polyhedron (in three-dimensional space) or polygon (in two-dimensional space) of a point form is dual to the polyhedron or polygon of the corresponding face form, where 'dual' means that they have the same number of edges but the number of faces and vertices is interchanged (ITA10; Hahn, 2005). ${ }^{1}$ The eigensymmetry of a form is a point group $E$ which either coincides with the generating point group $\mathrm{P}$ or is a supergroup of it. We treat here specifically face forms, which build the morphology of a euhedral crystal, to emphasize some inconsistencies occurring in the literature.

The number of possible forms of a point group is infinite. In fact, symbols with two - such as $\{h k 0\}$ - or three general Miller indices $-\{h k l\}$ - actually correspond to an infinite number of forms, for the various possible values of the Miller indices. In a real crystal only a limited number of forms are actually realized, those with small Miller indices being typically of larger size. Forms are classified into a finite number of types, in terms of the Wyckoff positions of the point groups.

Face forms are classified on the basis of their symmetry properties and of their orientation with respect to the symmetry elements of the point groups in which they occur. Of particular relevance for our discussion is the classification on the basis of the eigensymmetry of the form. A form is called

\footnotetext{
${ }^{1}$ For the sake of brevity, the different chapters of International Tables for Crystallography will be quoted herein as ITA $X$ for Vol. A, or ITB $X$ for Vol. B, where $X$ is the number of the chapter.
}

characteristic if its eigensymmetry $\mathrm{E}$ coincides with the generating point group $\mathrm{P}$ and non-characteristic when $\mathrm{E}$ is a proper supergroup of $\mathrm{P}$. Face forms bring a name dictated by their eigensymmetry (Table 1) and in the following will be called geometric (face) forms because they are defined in terms of the eigensymmetry of their stereographic poles. The number of forms is 47 and the minimal eigensymmetry a geometric form may possess is $m m 2$ or 222 (both of order 4).

Frequently in the literature, 48 forms are quoted because the dihedron is split into 'sphenoid' and 'dome' depending on whether it occurs in crystal class 2 or $m$ [concerning the difference between point groups, point-group types and geometric crystal classes, see Nespolo \& Souvignier (2009)]; the eigensymmetry is, however, $m m 2$ in both cases. The separation of the dihedron into a dome and a sphenoid implicitly means to take into account not only the eigensymmetry of the geometric form but also some physical property of the faces building it. Quite obviously, if such an option is adopted, it should be applied to all 47 geometric forms, not only one of them. In that case, the number of forms becomes much larger, as we are going to show. [The following analysis was inspired by an email exchange (28 November 2005) with Theo Hahn, reporting the opinion of Ellen Novack on the purely geometric nature of the crystal forms typically used in crystallography.]

As a criterion to introduce the consideration of the symmetry of the physical properties of the faces building a form we can take inspiration from the dome/sphenoid distinction. The symmetry operation relating the two faces is an operation of the first kind (rotation) in the case of a sphenoid but of the second kind (reflection) in the case of a dome. We can imagine assigning a handedness to each face, which is either preserved or reversed, depending on the nature of the symmetry operation. Thus, the sphenoid (crystal class 2) would be composed of two left (or two right) faces, while the dome (crystal class $m$ ) would be composed of one left and one right face. If the two faces of the form do not present a handedness, i.e. if they are achiral, one obtains the dihedron (crystal class $m m 2$ ): the achirality comes from the fact that the eigensymmetry of the faces contains a symmetry operation of the second kind (mirror reflection; the stereographic poles of the faces are on a mirror). Accordingly, the three forms that split from a dihedron when the handedness is taken into account can be symbolically indicated as $\mathrm{L}^{2}, \mathrm{LR}$ and $\mathrm{A}^{2}$, where $\mathrm{L}, \mathrm{R}$ and $\mathrm{A}$ stand for left, right and achiral, respectively, and the superscript 2 indicates the number of faces with the indicated handedness. Obviously, $\mathrm{L}^{2}$ can equally be written as $\mathrm{R}^{2}$, thus leading to an enantiomorphic pair of sphenoids.

The same analysis applied to all 47 geometric crystal forms gives the results presented in Table 1, where they are split according to their handedness, represented by what we can call their handedness symbol, giving the sequence of the handedness of faces building the form. The handedness symbol is an immediate generalization of the symbols used for the analysis of the dihedron and is composed of sequences like $[\mathrm{LR}]^{n}$, where $n$ means $n$ repetitions of the faces to which the superscript $n$ is attached. For each form obtained in this way, 
Table 1

The 47 geometric face forms, split according to the handedness of the faces as determined by the geometric crystal classes where the forms occur.

The handedness symbol in the third column gives the handedness of the faces as A (achiral), L (left), R (right); the number of faces of the same type (A, L or R) occurring consecutively is given as an exponent (i.e. $\mathrm{L}^{4}$ is given instead of LLLL and [LR] $]^{2}$ instead of LRLR). In parentheses are the geometric crystal classes where the corresponding form occurs. Bold denotes the eigensymmetry of the geometric form, which is also the geometric crystal class where the form is characteristic. No distinction is made for the geometric crystal classes differing in their axial setting (like $\overline{4} 2 m$ versus $\overline{4} m 2$ or $31 m$ versus $3 m 1$ ) because this has no influence on either the eigensymmetry of the geometric form or the handedness of the faces. Geometric crystal classes with only symmetry operations of the first kind are compatible with the existence of an enantiomorphic pair of forms, of which only one representative (L) is given. Each geometric crystal class occurs only once for each form, with the exception of $\overline{4} 2 \mathrm{~m}$ and $2 / \mathrm{m}$ for the pinacoid, which may be oriented with the rotation axis of infinite order parallel or perpendicular to the twofold axis of the point group, producing a form of type LR or $\mathrm{A}^{2}$, respectively. Closed forms are described with the sequences of faces in the northern hemisphere first, starting from the doubly positive quadrant of the stereographic projection. A total of 130 crystallographic forms occur when the handedness of the faces is taken into account, and of these 66 give rise to 33 enantiomorphic pairs of which only the $\mathrm{L}$ variant is listed. The table thus gives the 97 affine forms, $i . e$. counting each enantiomorphic pair as one form, similar to the classification of space groups in 219 affine types, with only one representative for each of the 11 enantiomorphic pairs.

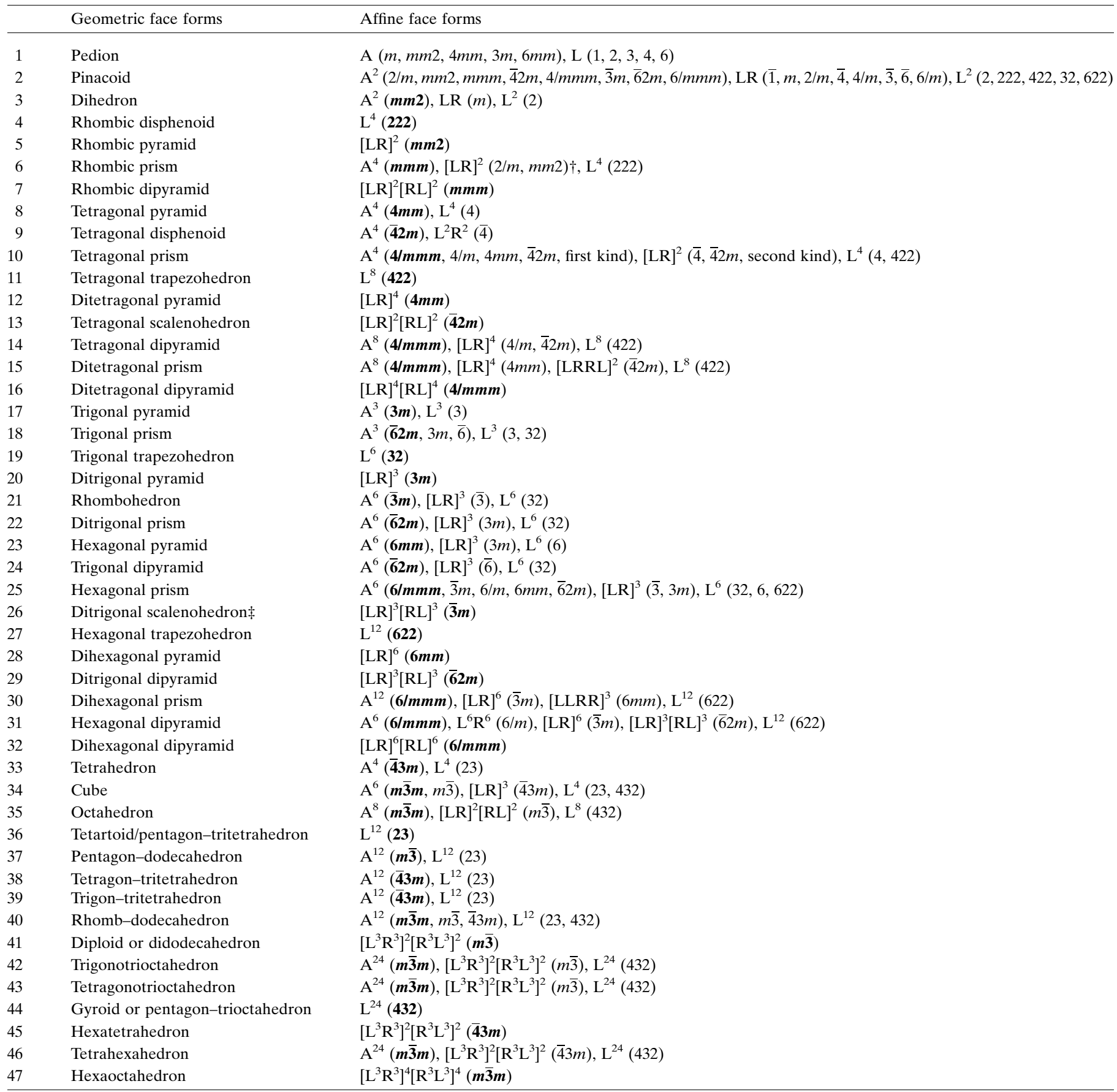

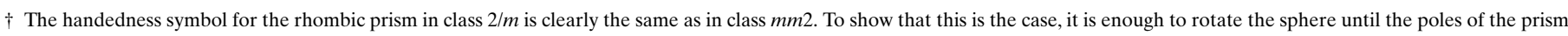

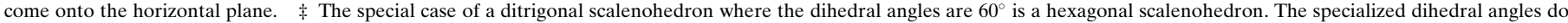
not increase the eigensymmetry of the form, as would be the case for a pyramid or dipyramid instead. 
the geometric crystal class(es) in which the form occurs is/are given in parentheses; bold indicates that the form is characteristic in that class, i.e. that the eigensymmetry of the form coincides with the point-group type of that class. Geometric crystal classes containing only operations of the first kind give rise to forms with faces of the same handedness, symbol $\mathrm{L}^{n}$. For each of them the form of the opposite handedness, $\mathrm{R}^{n}$, can also occur, giving birth to enantiomorphic pairs of forms.

Inspection of Table 1 reveals that the 47 geometric forms split into 97 affine forms. [The terms 'affine forms' and 'crystallographic forms' are introduced to follow the same criterion used to classify space groups into 219 affine types and 230 crystallographic types (ITA8).] Of these, 33 give rise to enantiomorphic pairs $\left(\mathrm{L}^{n}\right.$ and $\left.\mathrm{R}^{n}\right)$, to give a total of 130 crystallographic forms (no attempt to suggest specific names for each of them, like in the dome and sphenoid case, is made). It should thus be evident that the number of 48 found in some texts is spurious and finds its origin in the inconsistent consideration of the handedness of only one form.

Because the minimal eigensymmetry of a geometric form is orthorhombic, there are no characteristic forms in the triclinic and monoclinic crystal systems (ITA10). The morphology, at least if the crystal is euhedral, does reveal the point symmetry of the crystal, even in the lower-symmetry crystal systems. This comes from the fact that the morphological point symmetry of the whole crystalline edifice corresponds to the intersection of the eigensymmetries of the different forms building the crystal:

$$
\mathrm{P}=\cap_{i} \mathrm{E}(i),
$$

where $\mathrm{E}(i)$ is the eigensymmetry of the $i$ th form (geometric crystal class in bold in Table 1; the pedion and pinacoid are never characteristic and their eigensymmetries are $\infty m$ and $\infty m / m$, respectively).

The inconsistent terminology occurring sometimes in the literature has to be emphasized. In particular, the use of 'monoclinic prism' makes sense only with reference to an affine form. However, the morphological analysis is usually limited to the geometry of the sample, where no monoclinic prism exists, the corresponding geometric form having eigensymmetry $\mathrm{mmm}$.

If the term 'monoclinic prism' is not intrinsically wrong but simply often used inconsistently, the term 'triclinic prism' used by $\mathrm{Li}$ et al. (2010) is not correct, because a crystallographic prism is incompatible with the inversion centre as a unique symmetry element.

\section{Are Miller indices always coprime integers?}

The Miller indices of a family of lattice planes are usually presented as coprime integers, i.e. reduced of all common factors. This is correct when the direct lattice is indexed with respect to a primitive basis. However, to give the simplest possible matrix representation of the symmetry operations of a crystal it is customary to make reference to the so-called conventional cell (ITA9), which is often a centred cell. In this case, the use of coprime integers to represent a family of lattice planes, as done in many introductory texts, may become contradictory with respect to the definition itself of Miller indices. To show that this is the case, we have to recall that Miller indices represent the orientation of a crystal face (whether actually developed in the crystal morphology or not) and thus also of all the lattice planes parallel to it. The notation $(h k l)$ indicates a family of lattice planes, i.e. the whole set of lattice planes having the same orientation with respect to the chosen axial setting; the last of these planes, if actually developed in the crystal morphology, is the crystal face with the same indices. The Miller indices do not give the position of any of the lattice planes of a family, which is instead represented by the equation

$$
h x+k y+l z=n,
$$

(see equation 1.1.2.3 in ITB1; Shmueli, 2008) which precisely fixes the position as a function of the parameter $n$. Now, $n$ should take only integer values (ITB1) when the coordinates of a lattice node are put as the $x, y, z$ values in equation (2). In particular, it should give the first plane of the family after the origin, in the positive direction, when $n=1$. This plane thus has intercepts $1 / h, 1 / k$ and $1 / l$ on the $a, b$ and $c$ axes, respectively. When the unit cell is primitive or when the planes of the $(h k l)$ family are parallel to the face of the unit cell containing the centring vector, the above condition is consistent with coprime Miller integers. This is no longer true in the other cases. The contradiction is shown in Fig. 1 for the case of an $o P$ and an $o C$ type of unit cell and the family of planes $(h 00)$. The intercept on the $a$ axis of the first plane of the family is $1 / h$. If the unit cell is of type $P$ then the first plane after the origin passes through the lattice node 100 , so that $h=1$. In this case, the family of lattice planes has Miller indices (100). However, if the unit cell is of type $C$, the first plane after the origin passes through the lattice node $\frac{1}{2} \frac{1}{2} 0$, so that $h=2$. In this case, the

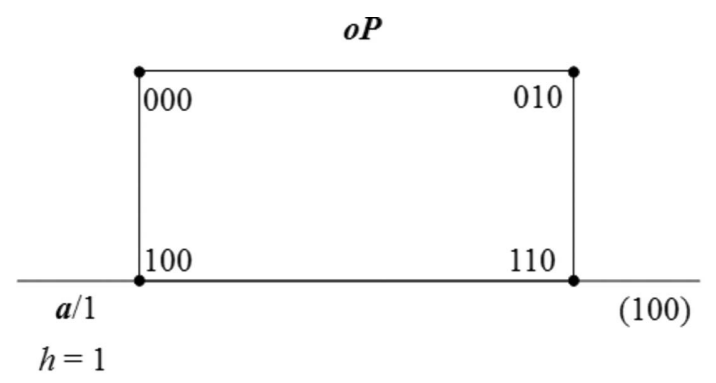

Figure 1

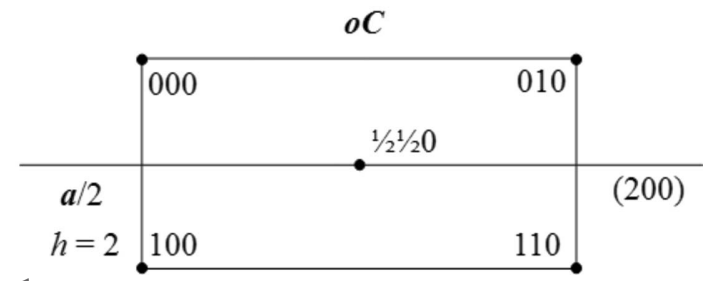

(Top) $o P$ and (bottom) $o C$ unit cells, seen in projection along [001]. The first plane of the $(h 00)$ family passes through the lattice node 100 in the case of $o P$ but through the lattice node $\frac{1}{2} \frac{1}{2} 0$ in the case of $o C$, so that the intersection with the $a$ axis is at 1 in the former but at $\frac{1}{2}$ in the latter. Consequently, $h$ is reduced to 1 and 2, respectively, and the two families of lattice planes are indicated as (100) and (200). 


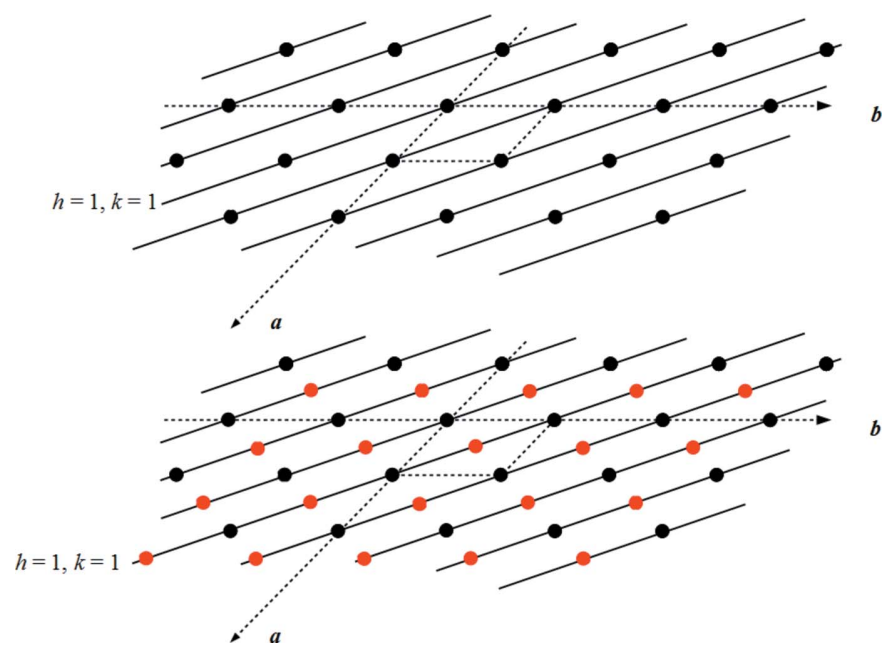

Figure 2

Part of the (001) section of a lattice whose conventional cell is of type $P$ (top) or $C$ (bottom), with the traces (solid lines) of ( $h k 0)$ planes with $h$ and $k$ having the same parity ( $h=k=1$ in this figure). The axes and boundaries of the conventional cell are drawn as dotted lines. Nodes centring the unit cell in $o C$ are shown in red to emphasize the difference between the two unit cells. The interplanar distance $d_{(h k 0)}$ is the same in the two lattices, but in $o C$ the density of nodes on each plane of the family is doubled with respect to $o P$. The first plane of the family passes through lattice nodes 100 and 010 in both cases, as well as through lattice node $\frac{1}{2} \frac{1}{2} 0$ in the case of $o C$, which in this family does not give rise to any additional plane with respect to $o P$. The same conclusions are obtained by drawing any other $(h k 0)$ family with the same parity of $h$ and $k$, like for example (310) or (150).

family of lattice planes has Miller indices (200). To call this family (100) would mean to state that the plane passing through lattice node 100 is the first of the family, while it is actually the second. In other words, it would mean denying the presence of the plane passing through $\frac{1}{2} \frac{1}{2} 0$, contradicting the choice of a $C$-centred unit cell. Also, putting the coordinates of lattice node $\frac{1}{2} \frac{1}{2} 0$ into equation (2) one obtains $m=\frac{1}{2}$ if the Miller indices of this family are given as (100). Quite obviously, the same happens for the family $(0 k 0)$ if the unit cell is $B$ centred and for the family $(00 l)$ if the unit cell is $A$ centred, and for all the three families simultaneously if the unit cell is $F$ centred. This result is immediately extended to the families $(h k 0)$ for a $C$-centred cell, $(h 0 l)$ for a $B$-centred cell and $(0 \mathrm{kl})$ for an $A$-centred cell: when the parity of the nonzero indices is the same, then the density of lattice planes is the same as that of a primitive unit cell, although the density of lattice nodes on each plane of the family is doubled (Fig. 2). ${ }^{2}$ In contrast, when the parity of the nonzero indices is opposite, the density of lattice planes is doubled with respect to that of a primitive unit cell, but the density of lattice nodes on each plane of the family is unchanged. Consequently, the first plane

\footnotetext{
2 These geometric considerations were already present by Friedel (1926) but applied to the computation of the twin index of twinned crystals [see details given by Nespolo \& Ferraris (2007)], while the effects on the Miller indices were not taken into account. The use of non-coprime integers for centred unit cells was proposed by Ungemach (1935) for the rhombohedral lattice and its generalization was proposed by Donnay (1936) in a somewhat obscure way, so that the first clear occurrence of this idea can be traced back to a footnote at p. 450 of the paper by Donnay \& Harker (1937).
}

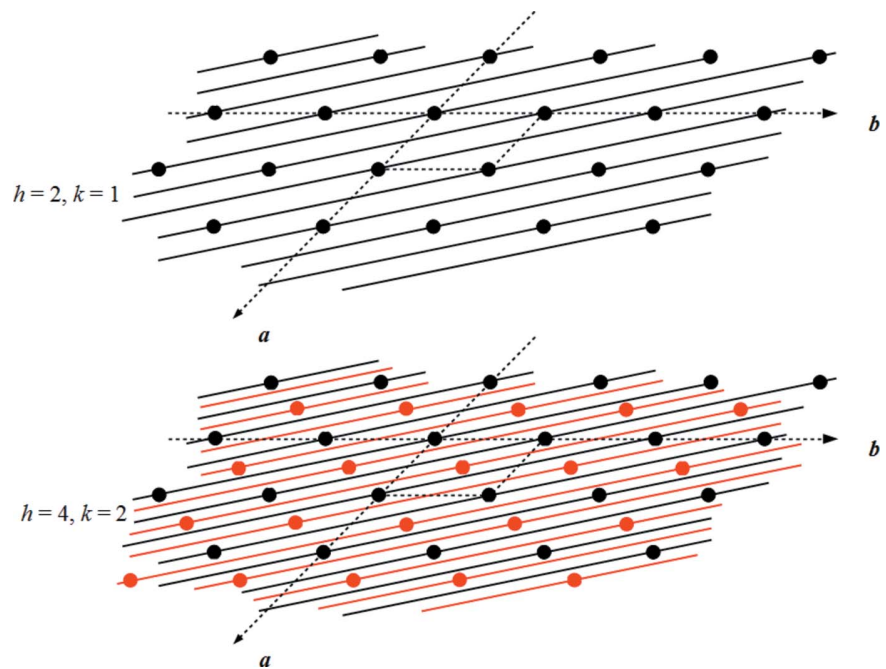

Figure 3

Part of the (001) section of a lattice whose conventional cell is of type $P$ (top) or $C$ (bottom), with the traces (solid lines) of ( $h k 0)$ planes with $h$ and $k$ having opposite parity ( $h=2, k=1$ in this figure). The same conventions are used as in Fig. 2. The interplanar distance $d_{(h k 0)}$ in $o C$ is half that in $o P$, but the density of nodes on each plane of the family is the same in both lattice types. The first plane of the family indeed cuts the $a$ axis at $\frac{1}{2}$ and passes through lattice node 010 in $o P$, so that the family of lattice planes is correctly expressed as (210). In the case of $o C$, however, there is an additional plane (red) midway between the origin and the plane which was first in $o P$; it cuts the $a$ axis at $\frac{1}{4}$ and the $b$ axis at $\frac{1}{2}$, so that the family of lattice planes is indexed as (420). The same conclusions are obtained by drawing any other $(h k 0)$ family with the opposite parity of $h$ and $k$, like for example (320) or (140).

of the family does not correspond to coprime integers but to indices having 2 as common factor (Fig. 3).

These geometric considerations can be translated in an analytical way by simply putting the $u v w$ coordinates of a lattice node into equation (2). If the value $n$ obtained is fractional, then the $h k l$ indices chosen to represent the family of planes are not correct: if $n$ is fractional with denominator $p$, with $p$ integer, then there are $p-1$ planes between the origin and what had been assumed to be the first plane of the family. Consequently, the $h k l$ indices must be multiplied by a factor $p$. For example, in the case of Fig. 3, the family of planes indicated as (210) would lead to $n=\frac{1}{2}$ when the lattice node $\frac{1}{2} \frac{1}{2} 0$ is inserted into equation (2), and to $n=\frac{3}{2}$ when the lattice node $\frac{3}{2} \frac{1}{2} 0$ is used instead. If the family of lattice planes is indicated as (420), then the two lattice nodes above give $n=1$ and 3 , respectively, when used in equation (2). This analytical translation of the geometric considerations is useful to analyse cases which are more difficult to show in a simple twodimensional projection. For example, in the case of an $I$-centred unit cell, the lattice plane of the family (111) which passes through lattice node $\frac{1}{2} \frac{1}{2} \frac{1}{2}$ would give $n=\frac{3}{2}$ in equation (2), meaning that the lattice plane which intersects each axis on the first node is not the first of the family but the second. Thus, the correct Miller indices of the family of lattice planes are (222). In the same way, starting from hypothetical Miller indices (100), (110) and (111) and inserting the coordinates of the node $\frac{2}{3} \frac{1}{3} \frac{1}{3}$ occurring in the obverse setting of a rhombohedral lattice with respect to hexagonal axes, the results are $n=$ 
$\frac{4}{3}, 1$ and $\frac{2}{3}$, showing that the corresponding lattice planes are the third, first and third of the families, respectively. The Miller indices thus have to be modified as (300), (110) and (333).

One immediately sees the relation between the Miller indices correctly expressed to satisfy equation (2) and the integral reflection conditions in reciprocal space, for which the same restrictions on the $h k l$ value of the diffractions occur as on the Miller indices of the family of lattice planes.

3.1. When non-coprime Miller indices become an artefact: the Bravais-Friedel-Donnay-Harker law

Friedel (1907) introduced the so-called Bravais law which establishes a rough proportionality between the frequency of occurrence of a given face in the morphology of a crystal and the density of nodes on the lattice planes corresponding to that face, i.e. having the same Miller indices. This geometric rule is an approximation introduced to predict the growth shapes of crystals, where the ranking of an $\{h k l\}$ form is related to the thickness of its corresponding layer: the thicker the layer, the higher the morphological importance of the form. A number of exceptions remained unexplained, concerning in particular the absence or low frequency of occurrence of faces with small Miller indices, and thus high densities of nodes. To cope with those exceptions, Donnay \& Harker (1937) introduced an extension of the Bravais law, today generally known as the Bravais-Friedel-Donnay-Harker law, which represents an effort to complement purely reticular considerations with some atomistic aspects. Unfortunately, this extension was performed in a rather spurious way, introducing confusion between the lattice and the structure and leading to an unnecessary multiplication of Miller indices.

The idea behind the Donnay-Harker extension was to take into account the influence of screw axes and glide planes in non-symmorphic space groups on the frequency of occurrence of the forms. Symmorphic space groups are generated, apart from the lattice translations, by point-group operations; they may contain screw rotations and glide reflections too, but these are not generators, as is instead the case for nonsymmorphic space groups. On the other hand, screw axes and glide planes in non-symmorphic space groups do represent a fundamental symmetry characteristic of the structure and do not depend on the axial setting chosen, although their representation obviously does: the Miller indices of a family of lattice planes cannot depend on the structural symmetry.

Donnay \& Harker (1937) made screw axes and glide planes act on lattice nodes (termed 'lattice equipoints') to produce a separate set of ghost points (termed 'space group equipoints') which, although not being lattice nodes, were treated as such, leading to an artificial multiplication of the Miller indices. We have seen that non-coprime Miller indices come from the use of centred cells, which are by no means an intrinsic feature of the crystal structure but a convenient choice for the axial setting. The Bravais-Friedel-Donnay-Harker law is a useful artefact in that it improves the predictive power of the Bravais law on the relative importance of crystal faces, but it is still an artefact. The improvement in the 'morphological aspect', as was called the decreasing sequence of forms, comes from the indirect consideration of the structural symmetry. Structural theories on the relative development of crystal faces, like the PBC (periodic bond chain) theory (Hartman \& Perdok, 1955), were developed later, making this useful artefact outdated. For example, Sunagawa (2005) has shown that the PBC theory predicts the correct 'morphological aspect' of pyrite, without the requirement of inconsistently multiplying the Miller indices of the faces depending on the zonal and serial reflection conditions, as was proposed by Donnay \& Harker (1937) to explain the observed sequence of forms.

\section{Laue indices of Bragg peaks versus Miller indices of} families of lattice planes

The indices of a Bragg reflection from the set of parallel equidistant planes $(h k l)$ are called Laue indices and are indicated as $h k l$ without parentheses (ITA1). Bragg's law,

$$
n \lambda=2 d_{h k l} \sin \theta_{h k l},
$$

includes the order of diffraction $n$; it is sometimes neglected but it is of paramount importance. Let us remember that Bragg's law is a geometric interpretation, in terms of the reflection of X-rays by lattice planes, of what is actually a diffraction phenomenon described by the Laue equations. It is the positive interference of the X-rays 'reflected' by the various planes of the same family $(\mathrm{hkl})$ that results in a Bragg peak, bringing the Laue indices $h k l$. There is thus an obvious correspondence between the Laue indices $h k l$ and the Miller indices $(h k l)$. Bragg peaks along a direction of reciprocal space are characterized by a common factor $n$ which increases with distance from the reciprocal space origin (position of the direct beam) and this is precisely the order of diffraction in equation (3). The expression 'diffraction from the plane ( $n h$ $n k n l$ )', which occurs more often than one might imagine, is at odds with both the definition of Miller indices and Bragg's law. In fact, if the Miller indices of a family of planes are $(h k l)$, not necessarily coprime integers (as we have seen above), they represent a family whose first plane after the origin of the axes has intercepts $\mathbf{a} / h, \mathbf{b} / k, \mathbf{c} / l$. A hypothetical family $(n h n k n l)$ would have the same orientation but its first plane would have intercepts $\mathbf{a} / n h, \mathbf{b} / n k, \mathbf{c} / n l$, which do not pass through any lattice node. In other words, the hypothetical family ( $n h n k n l)$ would include $n-1$ planes out of $n$ which are not reticular planes (do not pass through any lattice node). On the contrary, if these were reticular planes, then $(h k l)$ would not be a family of lattice planes, because it would be composed of only $1 / n$ of the planes with the same orientation. Therefore, the Laue indices $n h n k n l$ do not represent the first-order diffraction from a family ( $n h n k n l)$ but the $n$th order diffraction from the family $(h k l)$.

\section{Geometric derivation of the reflection conditions}

Most modern textbooks introduce the reflection conditions in an analytical way, showing that the structure factor is systematically zero for some relations between the $h k l$ Laue 
indices of the diffractions. While this is perfectly legitimate and absolutely correct, from the pedagogical viewpoint it is questionable as the best way to present the reflection conditions, especially for beginners who may not yet have experience of diffraction experiments. A geometric approach, proposed a long time ago by Buerger (1942) but today often forgotten (although mentioned in ITA2), is preferable in that it gives a much more concrete image and allows an easy memorization of the reflection conditions, without the need for an analytical derivation of the annihilation conditions for the structure factor. This presentation makes use of notions and concepts in direct space that the student has already (hopefully!) mastered and it is easier to understand, in the same way as the use of the polar lattice is pedagogically useful when introducing the reciprocal lattice (Nespolo \& Souvignier, 2010).

In $\S 2$ we have seen that a straightforward relation exists between the indexing of lattice planes and the integral reflection conditions, which are purely geometric in nature; they find their origin in the choice of the axial setting used to describe the crystal structure and are common to all space groups, both symmorphic and non-symmorphic. The specialization of the integral reflection conditions to two- and onedimensional sections (i.e. obtained by zeroing one or two indices) has no structural origin, as shown by the fact that the use of a primitive non-conventional unit cell would completely annihilate the integral reflection conditions, including those on these sections. On the other hand, non-symmorphic space groups show zonal and/or serial reflection conditions, coming from generating glide reflections and screw rotations, respectively; they do not disappear if a different unit cell is chosen, although their expression obviously depends on the axial setting chosen.

\subsection{Zonal reflection conditions}

Zonal reflection conditions come from the presence of glide planes. Fig. 4 shows the effect of an $a$ glide perpendicular to the [001] direction, through the example of an orbit $2 e$ in a space group of type Pmma. When the figure is observed in projection along this same direction, the distinction between atoms above and below the plane of the drawing is lost so that in this projection the periodicity along $\mathbf{a}$ is apparently halved. Because of the dual relation between the two spaces, the opposite is true in reciprocal space, where the periodicity along $\mathbf{a}^{*}$ is apparently doubled when a projection along [001] is observed. Accordingly, on the plane that is perpendicular to [001], i.e. the (001)* plane, one position out of two along $\mathbf{a}^{*}$ seems to be missing. Expressed formally, this results in the well known reflection condition $h k 0: h=2 n$. Obviously, by taking a $b$ glide instead of an $a$ glide, the reflection conditions become $h k 0: k=2 n$, and by taking another direction of projection, the corresponding zonal reflection conditions for $h 0 l$ and $0 \mathrm{kl}$ are obtained.

The case of a diagonal glide is shown in Fig. 5 for the case of an $n$ glide perpendicular to [11̄0], which occurs in cubic space groups ( $P n \overline{3} n$ and $P m \overline{3} n$, Nos. 222 and 223). The plane of the
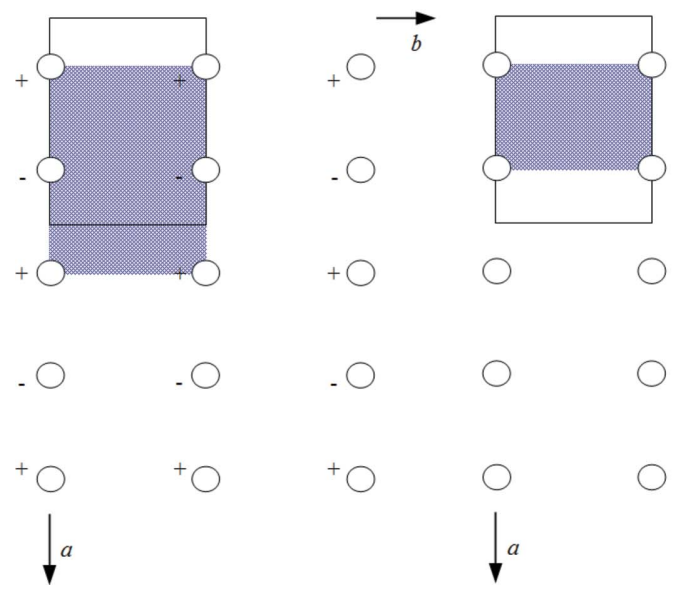

Figure 4

(Left) Part of the $2 e$ orbit $\left(\frac{1}{4} 0 z, \frac{3}{4} 0 \bar{z}\right)$ of a space group of type Pmma (No. 51 , standard setting) and (right) its projection on the (001) plane. The height ( $z$ coordinate) is shown as + and - , as is customary in ITA7. No chirality ('comma') is shown on the atoms because the site-symmetry group of Wyckoff position $2 e$ is $m m 2$. The hatched area on the left-hand side of the figure is the unit cell shifted by $a / 4$ on an atom, to emphasize that the orbit has the same translation vectors as the space group (i.e. it is not an extraordinary orbit). The right-hand side of the figure, obtained by projection so that all the atoms appear on the plane and no distinction by height appears, shows that in this projection the additional translation vector $\frac{1}{2} 00$ appears, so that the unit cell seems to be halved along the $a$ axis.

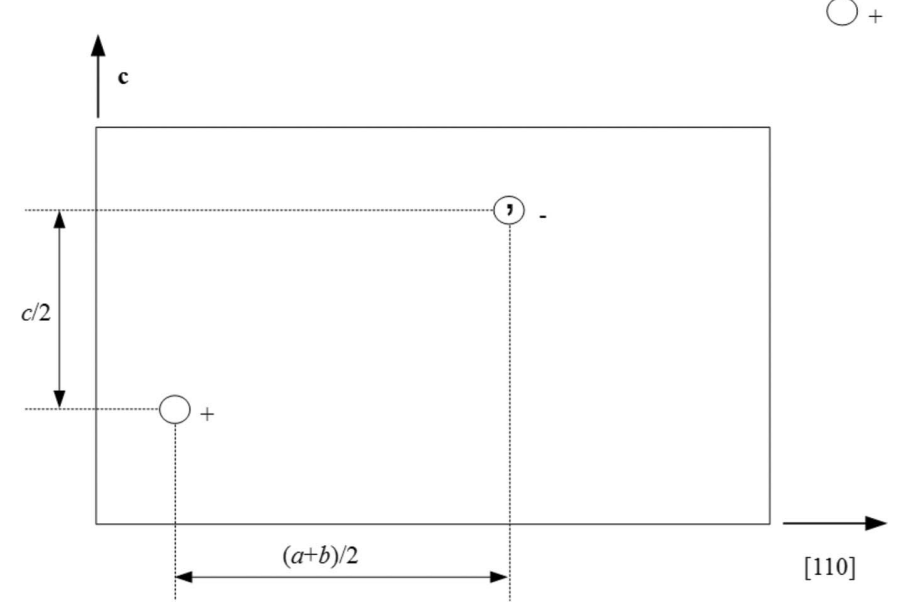

Figure 5

The effect of an $n$ glide perpendicular to the [11̄0] direction. Atoms on the opposite side of the glide plane (differentiated by the + and - signs, indicating the different heights along the [11̄0] direction, as well as by the opposite handedness) are separated by a vector $(\mathbf{a}+\mathbf{b}+\mathbf{c}) / 2$ in projection on the $(h h l)$ plane. When observed in projection, the height is lost and an apparent half-periodicity along the diagonal of the plane is observed. The same figure applies also to a $d$ glide by simply replacing the factor 2 by 4 .

projection is $(1 \overline{1} 0)$ and contains the directions [110] and [001]. The glide component is half of the in-plane horizontal direction, i.e. $(\mathbf{a}+\mathbf{b}) / 2$, and half of the in-plane vertical direction, i.e. $\mathbf{c} / 2$, so that the glide component is $(\mathbf{a}+\mathbf{b}+\mathbf{c}) / 2$. In the [110] projection, the periodicity along this direction is apparently halved. In reciprocal space, the plane perpendicular to $[1 \overline{1} 0]$ is $(1 \overline{1} 0)^{*}$ and in the projection on this plane the periodicity along the diagonal, which is $h+h+l$, is apparently doubled. The reflection condition for an $n$ glide perpendicular to [1ํㅣㄹ is 
therefore $h h l: 2 h+l=2 n$. However, because $2 h$ is always an even number, the condition $2 h+l=2 n$ gives the same result as $l=2 n$, and this is the way in which the reflection conditions are presented in ITA 3 and ITA7. If the glide plane is of type $d$ (which for the chosen orientation occurs in tetragonal and cubic space groups), the apparent periodicity in direct space is reduced to one-quarter, and in reciprocal space it is four times bigger, so that the reflection condition becomes $h h l: 2 h+l=4 n$.

\subsection{Serial reflection conditions}

Serial reflection conditions come from the presence of screw axes. Fig. 6 shows the effect of a $4_{1}$ screw axis parallel to the [001] direction. When the figure is observed in projection on this same direction, all the atoms around the axis are projected onto the axis, so that in this projection the periodicity along $\mathbf{c}$ is apparently reduced to one-quarter. Because of the dual relation between the two spaces, the opposite is true in reciprocal space, where the periodicity along $\mathbf{c}^{*}$ is apparently four times larger when a projection on [001]* is observed. Accordingly, three positions out of four along $\mathbf{c}^{*}$ seem to be missing. Expressed formally, this results in the well known reflection condition 00l: $l=4 n$. The same geometric reasoning applies to all the screw axes.

\section{Can the lack of symmetry impose restrictions?}

A number of recent (and not so recent) textbooks introduce the concept of crystal system on the basis of the metric rela-

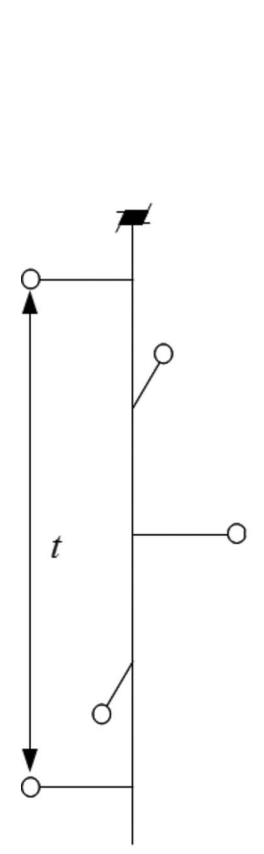

(a)

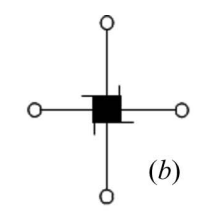

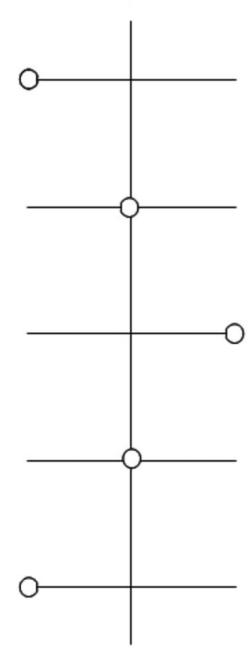

(c)

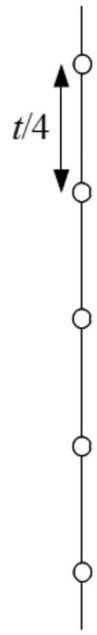

$(d)$
Figure 6

Effect of a $4_{1}$ screw axis parallel to the $c$ axis. (a) A perspective view, where $t$ is the full translation. (b) A projection along the $c$ axis; the screw component is lost. (c) A projection on the (100) or (010) plane. (d) A projection on the [001] axis. In this last, an apparent periodicity of $t / 4$ occurs. tions of the conventional unit cell, which is incorrect. A crystal system (shortened wording for crystal-class system) contains a complete set of geometric crystal classes of space groups (ITA8). The definition of a crystal system is thus based on the presence of a minimal set of point symmetry operations that are common to all the crystals belonging to the same crystal system. The symmetry of the conventional unit cell may, and often does, coincide with the holohedry of the crystal, but it may also be higher, in which case one speaks of accidental higher metric symmetry, which occurs more frequently than one might expect (Janner, 2004a,b). While the presence of a structural symmetry operation does impose restrictions on the metric of the unit cell, the absence of it cannot impose any restriction. Thus, in a monoclinic crystal, the presence of a twofold axis, or of a mirror perpendicular to it (or of both, of course), imposes the metric restriction of two right angles, which are chosen as $\alpha$ and $\gamma$ if the crystal is referred to the conventional $b$-unique setting. However, these operations do not have any influence on the value of the third angle ( $\beta$ in the conventional $b$-unique setting), which is symmetry unrestricted. It may take any value, and vary as a function of the external conditions (temperature $T$, pressure $p$ ), including $90^{\circ}$ as a special case, at least in a certain interval of $T$ and $p$ and within the standard uncertainty of the experimental determination. To define a monoclinic crystal as having $\beta \neq 90^{\circ}$ is incorrect because no metric restriction can occur as a consequence of the lack of symmetry. Again, to state that in a monoclinic crystal $a \neq b \neq c$ is incorrect for the same reasons.

The situation is different if one makes reference to the lattice, thus making abstraction from the structure. A specialized metric does promote the lattice to a higher symmetry, but without increasing the symmetry of the structure. Because of the possible divergence between lattice symmetry and structural symmetry, the concept of a Bravais flock is used in ITA8 to assign space groups (which represent the structural symmetry) to the Bravais class not affected by the accidental metric symmetry. However also in this case, the metric restrictions given in most textbooks are incorrect: they are too restrictive in some cases and not enough in others. The metric restrictions on the lattices are in fact much more complex than those on the structure, because one has to avoid all special cases, i.e. the specializations of the metric that promote the lattice symmetry to a higher holohedry. These specializations have recently been reviewed in great detail by Grimmer (2015); we give here just one example to emphasize how misleading are the metric restrictions frequently found in textbooks.

Let us consider the example of the metric restrictions for a monoclinic lattice, which are often given as $a \neq b \neq c ; \alpha=\gamma=$ $90^{\circ}, \beta \neq 90^{\circ}$, expressed in the common $b$-unique setting. The condition on the $b$ parameter is unnecessary. In fact, even in the case of a specialized metric with $a=b$ or with $b=c$, the symmetry of the lattice is not promoted to a higher holohedry, unless further specializations exist too. On the other hand, to exclude only the $90^{\circ}$ for $\beta$ is not enough. If the cell parameters define a primitive unit cell and $\beta=\cos ^{-1}(-a / 2 c)$, then the lattice is actually $o B$ (orthorhombic with a $B$-centred cell, 
Table 2

Metric restrictions on the crystal system imposed by the symmetry of the crystal.

\begin{tabular}{ll}
\hline Crystal system & Metric restrictions \\
\hline Triclinic & None \\
Monoclinic ( $b$-unique) & $\alpha=\gamma=90^{\circ}$ \\
Orthorhombic & $\alpha=\beta=\gamma=90^{\circ}$ \\
Tetragonal & $a=b ; \alpha=\beta=\gamma=90^{\circ}$ \\
Trigonal, rhombohedral axes & $a=b=c ; \alpha=\beta=\gamma$ \\
Trigonal and hexagonal, hexagonal axes & $a=b ; \alpha=\beta=90^{\circ}, \gamma=120^{\circ}$ \\
Cubic & $a=b=c ; \alpha=\beta=\gamma=90^{\circ}$ \\
\hline
\end{tabular}

which can be transformed to $O C$ or $O A$ by a permutation of the axes). If the cell parameters define a body-centred unit cell and $\beta=\cos ^{-1}(-a / c)$, then the lattice is actually $o C$; if $a^{2}+b^{2}=$ $c^{2}$ and $a^{2}+a c \cos \beta=b^{2}$ or $b^{2}+a c \cos \beta=a^{2}$, then the lattice is actually $h R$ (rhombohedral); if $c^{2}+3 b^{2}=9 a^{2}$ and $c=-3 a \cos \beta$ or $a^{2}+3 b^{2}=9 c^{2}$ and $a=-3 c \cos \beta$, then the lattice is again $h R$ (rhombohedral) (data from Table 9.4.3.1 in ITA9; unit cells of type $m I, m C, m A$ and $m F$ are all easily converted into each other so that the above conditions for $m I$ can be transformed into the setting of any of the other three types of unit cell; see also Grimmer, 2015).

It should therefore be evident that, if one wants to give the metric restrictions for the lattices, the situation is much more complex than that given in any textbook. On the other hand, if the metric restrictions are those imposed by the structural symmetry, any accidentally specialized value which would increase the symmetry of the lattice should not be excluded. Therefore, when giving the metric relations imposed by the symmetry of the crystal, the correct relations are those shown in Table 2.

\section{Conclusions}

The time and space devoted to teaching fundamental crystallography in undergraduate programmes, especially in direct space, has been reduced drastically during the past few decades and it is still shrinking. One of the consequences is that some basic aspects are no longer presented, while others are covered very quickly in an approximate way that often leads to imprecisions, axiomatic statements and less effective presentations. This also occurs in textbooks, where the authors sometimes seem to feel obliged to insert some basic topics without which the text could be considered incomplete, but cannot devote enough space to a convincing presentation, so that the result is quite often less satisfactory than that already available in older classical textbooks.

We have presented a few examples of problems frequently and repeatedly seen in modern textbooks and class presentations and we have pointed out how a consistent and correct presentation can instead be realized by very simple, essentially geometric, considerations. We hope that this effort may be useful to avoid mistakes and increase the effectiveness of this basic part of the crystallography curriculum and contribute to restoring its charm, today unreasonably considered old fashioned.

\section{Acknowledgements}

This article was prepared during a stay at Tokyo Institute of Technology as Foreign Guest Professor. Comments from four anonymous reviewers, Professor Hans Grimmer and the Coeditor are gratefully acknowledged.

\section{References}

Buerger, M. J. (1942). X-ray Crystallography: An Introduction to the Investigation of Crystals by Their Diffraction of Monochromatic $X$-Radiation. New York: Wiley.

Donnay, J. D. H. (1936). Am. Mineral. 21 (No. 12, Part 2), 4-5.

Donnay, J. D. H. \& Harker, D. (1937). Am. Mineral. 22, 446-467.

Friedel, G. (1907). Bull. Soc. Franç. Mineral. 30, 326-455.

Friedel, G. (1926). Leçons de Cristallographie. Nancy, Paris, Strasbourg: Berger-Levrault.

Grimmer, H. (2015). Acta Cryst. A71, 143-149.

Hahn, Th. (2005). Editor. International Tables for Crystallography, Vol. A, Space-Group Symmetry, 5th ed. Heidelberg: Springer.

Hartman, P. \& Perdok, W. G. (1955). Acta Cryst. 8, 49-52.

Janner, A. (2004a). Acta Cryst. A60, 198-200.

Janner, A. (2004b). Acta Cryst. A60, 611-620.

Leopardi, G. (1836). La Ginestra, o il Fiore del Deserto. (The Broom, or the Flower of the Desert.) In Poems, translated by J. Galassi (2012). New York: Farrar, Straus and Giroux.

Li, L., Zhang, Y., Esling, C., Jiang, H., Zhao, Z., Zuo, Y. \& Cui, J. (2010). J. Appl. Cryst. 43, 1108-1112.

Nespolo, M. \& Ferraris, G. (2007). Acta Cryst. A63, 278-286.

Nespolo, M. \& Souvignier, B. (2009). Z. Kristallogr. 224, 127-136.

Nespolo, M. \& Souvignier, B. (2010). J. Appl. Cryst. 43, 1144-1149.

Schmueli, U. (2008). Editor. International Tables for Crystallography, Vol. B, Reciprocal Space, 3rd ed. Heidelberg: Springer.

Sunagawa, I. (2005). Crystals. Growth, Morphology and Perfection. Cambridge University Press.

Ungemach, H. (1935). Z. Kristallogr. 91, 97-113. 